Supplement of Hydrol. Earth Syst. Sci., 22, 5759-5779, 2018

https://doi.org/10.5194/hess-22-5759-2018-supplement

(C) Author(s) 2018. This work is distributed under

the Creative Commons Attribution 4.0 License.

(c) (1)

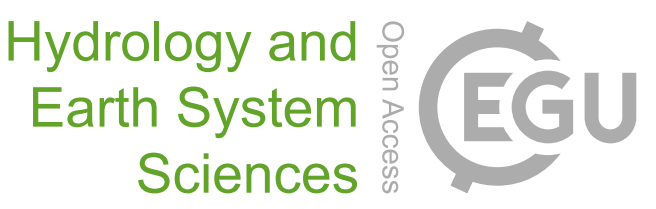

Supplement of

\title{
Hybridizing Bayesian and variational data assimilation for high-resolution hydrologic forecasting
}

Felipe Hernández and Xu Liang

Correspondence to: Xu Liang (xuliang@pitt.edu)

The copyright of individual parts of the supplement might differ from the CC BY 4.0 License. 


\section{Supplementary information}

Table S1. Parameter assignments and error metrics for all the configurations of Experiment 1 (Blue River), Scenario 1 (October $\left.15^{\text {th }}, 1996\right)$. Configuration 0 corresponds to the control open-loop model. All other errors are computed using the mean streamflow from the OPTIMISTS ensemble.

\begin{tabular}{|c|c|c|c|c|c|c|c|c|c|c|c|}
\hline \multirow{2}{*}{ Config. } & \multirow{2}{*}{ Objs. } & \multirow{2}{*}{$n$} & \multirow{2}{*}{$\Delta t$} & \multirow{2}{*}{$p_{\text {samp }}$} & \multirow{2}{*}{$k_{\text {F-class }}$} & \multicolumn{3}{|c|}{ Assimilation period } & \multicolumn{3}{|c|}{ Forecast period } \\
\hline & & & & & & $\mathrm{NSE}_{\mathfrak{2} 2}$ & $\mathrm{NSE}_{\ell 1}$ & MARE & $\mathrm{NSE}_{\ell 2}$ & $\mathrm{NSE}_{\boldsymbol{t} 1}$ & MARE \\
\hline 0 & & No de & assil & ilation & - & 0.778 & 0.659 & $35.8 \%$ & 0.867 & 0.708 & $43.2 \%$ \\
\hline 1 & 1 & 100 & $1 \mathrm{~d}$ & 0.3 & false & 0.640 & 0.545 & $572.6 \%$ & 0.494 & 0.395 & $118.5 \%$ \\
\hline 2 & 1 & 100 & $1 \mathrm{~d}$ & 0.3 & true & 0.811 & 0.661 & $383.8 \%$ & 0.818 & 0.656 & $53.2 \%$ \\
\hline 3 & 1 & 100 & $1 \mathrm{~d}$ & 1.0 & false & 0.796 & 0.670 & $25.2 \%$ & 0.722 & 0.630 & $43.9 \%$ \\
\hline 4 & 1 & 100 & $1 \mathrm{~d}$ & 1.0 & true & 0.791 & 0.692 & $29.9 \%$ & 0.707 & 0.564 & $50.4 \%$ \\
\hline 5 & 1 & 100 & $5 \mathrm{~d}$ & 0.3 & false & 0.923 & 0.790 & $19.0 \%$ & 0.770 & 0.645 & $38.5 \%$ \\
\hline 6 & 1 & 100 & $5 \mathrm{~d}$ & 0.3 & true & 0.902 & 0.774 & $20.5 \%$ & 0.842 & 0.696 & $38.1 \%$ \\
\hline 7 & 1 & 100 & $5 \mathrm{~d}$ & 1.0 & false & 0.791 & 0.670 & $24.5 \%$ & 0.773 & 0.650 & $42.6 \%$ \\
\hline 8 & 1 & 100 & $5 \mathrm{~d}$ & 1.0 & true & 0.849 & 0.731 & $20.3 \%$ & 0.593 & 0.542 & $50.8 \%$ \\
\hline 9 & 1 & 100 & $2 w$ & 0.3 & false & 0.801 & 0.668 & $46.0 \%$ & 0.853 & 0.690 & $44.5 \%$ \\
\hline 10 & 1 & 100 & $2 \mathrm{w}$ & 0.3 & true & 0.827 & 0.690 & $41.9 \%$ & 0.823 & 0.687 & $42.1 \%$ \\
\hline 11 & 1 & 100 & $2 \mathrm{w}$ & 1.0 & false & 0.802 & 0.671 & $46.5 \%$ & 0.860 & 0.700 & $44.1 \%$ \\
\hline 12 & 1 & 100 & $2 \mathrm{w}$ & 1.0 & true & 0.838 & 0.696 & $45.2 \%$ & 0.864 & 0.702 & $44.0 \%$ \\
\hline 13 & 1 & 500 & $1 \mathrm{~d}$ & 0.3 & false & $-1 E+2$ & $-5 E+0$ & $2 \mathrm{E}+1$ & -0.331 & 0.133 & $92.0 \%$ \\
\hline 14 & 1 & 500 & $1 \mathrm{~d}$ & 0.3 & true & $-5 E+2$ & $-2 \mathrm{E}+1$ & $6 \mathrm{E}+1$ & $-2 \mathrm{E}+1$ & -2.388 & $863.1 \%$ \\
\hline 15 & 1 & 500 & $1 \mathrm{~d}$ & 1.0 & false & 0.823 & 0.687 & $25.9 \%$ & 0.771 & 0.657 & $43.3 \%$ \\
\hline 16 & 1 & 500 & $1 \mathrm{~d}$ & 1.0 & true & 0.808 & 0.684 & $40.5 \%$ & 0.698 & 0.553 & $55.2 \%$ \\
\hline 17 & 1 & 500 & $5 \mathrm{~d}$ & 0.3 & false & 0.789 & 0.654 & $40.0 \%$ & 0.787 & 0.625 & $48.2 \%$ \\
\hline 18 & 1 & 500 & $5 \mathrm{~d}$ & 0.3 & true & 0.791 & 0.632 & $161.4 \%$ & 0.533 & 0.518 & $37.5 \%$ \\
\hline 19 & 1 & 500 & $5 \mathrm{~d}$ & 1.0 & false & 0.810 & 0.684 & $23.5 \%$ & 0.847 & 0.697 & $42.3 \%$ \\
\hline 20 & 1 & 500 & $5 \mathrm{~d}$ & 1.0 & true & 0.723 & 0.621 & $27.4 \%$ & 0.675 & 0.584 & $46.4 \%$ \\
\hline 21 & 1 & 500 & $2 \mathrm{w}$ & 0.3 & false & 0.851 & 0.713 & $86.7 \%$ & 0.875 & 0.729 & $36.0 \%$ \\
\hline 22 & 1 & 500 & $2 \mathrm{w}$ & 0.3 & true & 0.808 & 0.680 & $71.9 \%$ & 0.801 & 0.652 & $38.2 \%$ \\
\hline 23 & 1 & 500 & $2 \mathrm{w}$ & 1.0 & false & 0.819 & 0.685 & $43.7 \%$ & 0.850 & 0.704 & $43.4 \%$ \\
\hline 24 & 1 & 500 & $2 w$ & 1.0 & true & 0.845 & 0.699 & $42.9 \%$ & 0.844 & 0.698 & $43.5 \%$ \\
\hline 25 & 2 & 100 & $1 \mathrm{~d}$ & 0.3 & false & 0.826 & 0.699 & $188.4 \%$ & 0.856 & 0.711 & $37.7 \%$ \\
\hline 26 & 2 & 100 & $1 \mathrm{~d}$ & 0.3 & true & 0.850 & 0.684 & $142.3 \%$ & 0.836 & 0.618 & $82.2 \%$ \\
\hline
\end{tabular}




\begin{tabular}{|c|c|c|c|c|c|c|c|c|c|c|c|}
\hline \multirow{2}{*}{ Config. } & \multirow{2}{*}{ Objs. } & \multirow{2}{*}{$n$} & \multirow{2}{*}{$\Delta t$} & \multirow{2}{*}{$p_{\text {samp }}$} & \multirow{2}{*}{$k_{\mathrm{F} \text {-class }}$} & \multicolumn{3}{|c|}{ Assimilation period } & \multicolumn{3}{|c|}{ Forecast period } \\
\hline & & & & & & $\mathrm{NSE}_{\ell 2}$ & $\mathrm{NSE}_{\ell 1}$ & MARE & $\mathrm{NSE}_{\ell 2}$ & $\mathrm{NSE}_{\ell 1}$ & MARE \\
\hline 27 & 2 & 100 & $1 \mathrm{~d}$ & 1.0 & false & 0.762 & 0.640 & $29.5 \%$ & 0.809 & 0.667 & $45.7 \%$ \\
\hline 28 & 2 & 100 & $1 \mathrm{~d}$ & 1.0 & true & 0.783 & 0.659 & $29.5 \%$ & 0.833 & 0.639 & $55.8 \%$ \\
\hline 29 & 2 & 100 & $5 \mathrm{~d}$ & 0.3 & false & 0.837 & 0.708 & $25.8 \%$ & 0.827 & 0.680 & $44.6 \%$ \\
\hline 30 & 2 & 100 & $5 \mathrm{~d}$ & 0.3 & true & 0.868 & 0.750 & $19.1 \%$ & 0.486 & 0.509 & $47.4 \%$ \\
\hline 31 & 2 & 100 & $5 \mathrm{~d}$ & 1.0 & false & 0.735 & 0.633 & $30.6 \%$ & 0.831 & 0.687 & $43.3 \%$ \\
\hline 32 & 2 & 100 & $5 \mathrm{~d}$ & 1.0 & true & 0.776 & 0.657 & $26.0 \%$ & 0.866 & 0.710 & $43.0 \%$ \\
\hline 33 & 2 & 100 & $2 \mathrm{w}$ & 0.3 & false & 0.785 & 0.663 & $42.8 \%$ & 0.852 & 0.706 & $42.0 \%$ \\
\hline 34 & 2 & 100 & $2 \mathrm{w}$ & 0.3 & true & 0.792 & 0.666 & $41.4 \%$ & 0.838 & 0.693 & $43.6 \%$ \\
\hline 35 & 2 & 100 & $2 \mathrm{w}$ & 1.0 & false & 0.777 & 0.656 & $43.5 \%$ & 0.857 & 0.702 & $43.6 \%$ \\
\hline 36 & 2 & 100 & $2 \mathrm{w}$ & 1.0 & true & 0.800 & 0.670 & $40.6 \%$ & 0.841 & 0.693 & $43.7 \%$ \\
\hline 37 & 2 & 500 & $1 \mathrm{~d}$ & 0.3 & false & 0.772 & 0.642 & $340.0 \%$ & 0.764 & 0.595 & $67.6 \%$ \\
\hline 38 & 2 & 500 & $1 \mathrm{~d}$ & 0.3 & true & 0.521 & 0.494 & $713.3 \%$ & 0.759 & 0.528 & $87.5 \%$ \\
\hline 39 & 2 & 500 & $1 \mathrm{~d}$ & 1.0 & false & 0.770 & 0.645 & $28.7 \%$ & 0.838 & 0.693 & $42.9 \%$ \\
\hline 40 & 2 & 500 & $1 \mathrm{~d}$ & 1.0 & true & 0.770 & 0.634 & $181.4 \%$ & 0.815 & 0.647 & $48.4 \%$ \\
\hline 41 & 2 & 500 & $5 \mathrm{~d}$ & 0.3 & false & 0.858 & 0.725 & $50.7 \%$ & 0.826 & 0.680 & $41.8 \%$ \\
\hline 42 & 2 & 500 & $5 \mathrm{~d}$ & 0.3 & true & 0.878 & 0.755 & $38.3 \%$ & 0.230 & 0.428 & $34.7 \%$ \\
\hline 43 & 2 & 500 & $5 \mathrm{~d}$ & 1.0 & false & 0.755 & 0.646 & $29.2 \%$ & 0.858 & 0.708 & $42.7 \%$ \\
\hline 44 & 2 & 500 & $5 \mathrm{~d}$ & 1.0 & true & 0.766 & 0.650 & $26.3 \%$ & 0.650 & 0.574 & $47.1 \%$ \\
\hline 45 & 2 & 500 & $2 \mathrm{w}$ & 0.3 & false & 0.860 & 0.718 & $45.4 \%$ & 0.873 & 0.718 & $38.6 \%$ \\
\hline 46 & 2 & 500 & $2 w$ & 0.3 & true & 0.881 & 0.745 & $60.7 \%$ & 0.873 & 0.720 & $41.2 \%$ \\
\hline 47 & 2 & 500 & $2 w$ & 1.0 & false & 0.799 & 0.672 & $42.8 \%$ & 0.868 & 0.708 & $43.2 \%$ \\
\hline 48 & 2 & 500 & $2 w$ & 1.0 & true & 0.793 & 0.670 & $43.4 \%$ & 0.864 & 0.707 & $43.3 \%$ \\
\hline
\end{tabular}

Table S2. Parameter assignments and error metrics for all the configurations of Experiment 1 (Blue River), Scenario 2 (January $15^{\text {th }}, 1997$ ). Configuration 0 corresponds to the control open-loop model. All other errors are computed using the mean streamflow 5 fhemPTIMISTS ensemble.

\begin{tabular}{|c|c|c|c|c|c|c|c|c|c|c|c|}
\hline \multirow{2}{*}{ Config. } & \multirow{2}{*}{ Objs. } & \multirow{2}{*}{$n$} & \multirow{2}{*}{$\Delta t$} & \multirow{2}{*}{$p_{\text {samp }}$} & \multirow{2}{*}{$k_{\text {F-class }}$} & \multicolumn{3}{|c|}{ Assimilation period } & \multicolumn{3}{|c|}{ Forecast period } \\
\hline & & & & & & $\mathrm{NSE}_{\ell 2}$ & $\mathrm{NSE}_{\ell 1}$ & MARE & $\mathrm{NSE}_{\ell 2}$ & $\mathrm{NSE}_{\ell 1}$ & MARE \\
\hline 0 & \multicolumn{5}{|c|}{-- No data assimilation -- } & 0.229 & 0.146 & $5.4 \%$ & -0.122 & 0.296 & $47.8 \%$ \\
\hline 1 & 1 & 100 & $1 \mathrm{~d}$ & 0.3 & false & $-3 E+6$ & $-1 E+3$ & $7 \mathrm{E}+1$ & 0.364 & 0.365 & $50.4 \%$ \\
\hline 2 & 1 & 100 & $1 \mathrm{~d}$ & 0.3 & true & $-1 E+6$ & $-5 E+2$ & $4 \mathrm{E}+1$ & $-1 E+1$ & -2.022 & $322.7 \%$ \\
\hline 3 & 1 & 100 & $1 \mathrm{~d}$ & 1.0 & false & 0.514 & 0.217 & $5.3 \%$ & -0.871 & 0.025 & $65.6 \%$ \\
\hline 4 & 1 & 100 & $1 \mathrm{~d}$ & 1.0 & true & 0.617 & 0.410 & $3.8 \%$ & 0.809 & 0.675 & $23.6 \%$ \\
\hline 5 & 1 & 100 & $5 \mathrm{~d}$ & 0.3 & false & 0.328 & 0.211 & $5.1 \%$ & 0.523 & 0.496 & $20.3 \%$ \\
\hline 6 & 1 & 100 & $5 \mathrm{~d}$ & 0.3 & true & 0.533 & 0.407 & $3.7 \%$ & 0.348 & 0.439 & $37.3 \%$ \\
\hline 7 & 1 & 100 & $5 \mathrm{~d}$ & 1.0 & false & 0.234 & 0.255 & $4.6 \%$ & 0.769 & 0.613 & $20.9 \%$ \\
\hline 8 & 1 & 100 & $5 \mathrm{~d}$ & 1.0 & true & 0.388 & 0.321 & $4.2 \%$ & 0.301 & 0.398 & $23.3 \%$ \\
\hline 9 & 1 & 100 & $2 w$ & 0.3 & false & 0.454 & 0.280 & $4.6 \%$ & 0.400 & 0.460 & $36.7 \%$ \\
\hline 10 & 1 & 100 & $2 \mathrm{w}$ & 0.3 & true & 0.468 & 0.241 & $4.8 \%$ & 0.092 & 0.346 & $44.4 \%$ \\
\hline 11 & 1 & 100 & $2 w$ & 1.0 & false & 0.512 & 0.341 & $4.2 \%$ & 0.500 & 0.478 & $35.4 \%$ \\
\hline 12 & 1 & 100 & $2 \mathrm{w}$ & 1.0 & true & 0.555 & 0.323 & $4.3 \%$ & 0.299 & 0.415 & $39.4 \%$ \\
\hline
\end{tabular}




\begin{tabular}{|c|c|c|c|c|c|c|c|c|c|c|c|}
\hline \multirow{2}{*}{ Config. } & \multirow{2}{*}{ Objs. } & \multirow{2}{*}{$n$} & \multirow{2}{*}{$\Delta t$} & \multirow{2}{*}{$p_{\text {samp }}$} & \multirow{2}{*}{$k_{\mathrm{F} \text {-class }}$} & \multicolumn{3}{|c|}{ Assimilation period } & \multicolumn{3}{|c|}{ Forecast period } \\
\hline & & & & & & $\mathrm{NSE}_{\ell 2}$ & $\mathrm{NSE}_{\ell 1}$ & MARE & $\mathrm{NSE}_{12}$ & $\mathrm{NSE}_{\ell 1}$ & MARE \\
\hline 13 & 1 & 500 & $1 \mathrm{~d}$ & 0.3 & false & $-7 \mathrm{E}+6$ & $-2 \mathrm{E}+3$ & $1 \mathrm{E}+2$ & -0.456 & 0.009 & $46.9 \%$ \\
\hline 14 & 1 & 500 & $1 \mathrm{~d}$ & 0.3 & true & $-2 \mathrm{E}+7$ & $-4 \mathrm{E}+3$ & $3 E+2$ & $-4 E+3$ & $-4 \mathrm{E}+1$ & $6 \mathrm{E}+1$ \\
\hline 15 & 1 & 500 & $1 \mathrm{~d}$ & 1.0 & false & 0.596 & 0.428 & $3.7 \%$ & -0.809 & -0.059 & $69.0 \%$ \\
\hline 16 & 1 & 500 & $1 \mathrm{~d}$ & 1.0 & true & 0.508 & 0.338 & $4.2 \%$ & 0.865 & 0.699 & $18.6 \%$ \\
\hline 17 & 1 & 500 & $5 \mathrm{~d}$ & 0.3 & false & 0.417 & 0.240 & $4.9 \%$ & 0.695 & 0.543 & $31.2 \%$ \\
\hline 18 & 1 & 500 & $5 \mathrm{~d}$ & 0.3 & true & 0.445 & 0.233 & $5.0 \%$ & 0.557 & 0.537 & $30.1 \%$ \\
\hline 19 & 1 & 500 & $5 \mathrm{~d}$ & 1.0 & false & 0.266 & 0.275 & $4.5 \%$ & 0.768 & 0.660 & $22.6 \%$ \\
\hline 20 & 1 & 500 & $5 \mathrm{~d}$ & 1.0 & true & 0.294 & 0.301 & $4.4 \%$ & 0.662 & 0.596 & $27.6 \%$ \\
\hline 21 & 1 & 500 & $2 \mathrm{w}$ & 0.3 & false & 0.654 & 0.469 & $3.3 \%$ & 0.733 & 0.622 & $20.1 \%$ \\
\hline 22 & 1 & 500 & $2 \mathrm{w}$ & 0.3 & true & 0.548 & 0.398 & $3.8 \%$ & 0.681 & 0.564 & $27.4 \%$ \\
\hline 23 & 1 & 500 & $2 \mathrm{w}$ & 1.0 & false & 0.468 & 0.309 & $4.3 \%$ & 0.337 & 0.426 & $39.2 \%$ \\
\hline 24 & 1 & 500 & $2 w$ & 1.0 & true & 0.613 & 0.392 & $3.8 \%$ & 0.675 & 0.570 & $28.1 \%$ \\
\hline 25 & 2 & 100 & $1 \mathrm{~d}$ & 0.3 & false & $-3 E+1$ & -2.297 & $21.9 \%$ & 0.249 & 0.353 & $45.4 \%$ \\
\hline 26 & 2 & 100 & $1 \mathrm{~d}$ & 0.3 & true & 0.032 & 0.113 & $5.8 \%$ & 0.504 & 0.534 & $32.3 \%$ \\
\hline 27 & 2 & 100 & $1 \mathrm{~d}$ & 1.0 & false & 0.399 & 0.242 & $4.9 \%$ & -0.328 & 0.130 & $56.8 \%$ \\
\hline 28 & 2 & 100 & $1 \mathrm{~d}$ & 1.0 & true & 0.317 & 0.360 & $3.9 \%$ & 0.351 & 0.443 & $20.8 \%$ \\
\hline 29 & 2 & 100 & $5 \mathrm{~d}$ & 0.3 & false & 0.004 & 0.071 & $5.8 \%$ & 0.842 & 0.681 & $20.5 \%$ \\
\hline 30 & 2 & 100 & $5 \mathrm{~d}$ & 0.3 & true & 0.371 & 0.211 & $5.0 \%$ & 0.732 & 0.634 & $21.4 \%$ \\
\hline 31 & 2 & 100 & $5 \mathrm{~d}$ & 1.0 & false & 0.129 & 0.181 & $5.1 \%$ & 0.767 & 0.623 & $25.2 \%$ \\
\hline 32 & 2 & 100 & $5 \mathrm{~d}$ & 1.0 & true & 0.245 & 0.262 & $4.6 \%$ & 0.839 & 0.697 & $19.9 \%$ \\
\hline 33 & 2 & 100 & $2 \mathrm{w}$ & 0.3 & false & 0.276 & 0.136 & $5.5 \%$ & 0.269 & 0.403 & $40.7 \%$ \\
\hline 34 & 2 & 100 & $2 \mathrm{w}$ & 0.3 & true & 0.571 & 0.352 & $4.1 \%$ & 0.507 & 0.491 & $34.3 \%$ \\
\hline 35 & 2 & 100 & $2 w$ & 1.0 & false & 0.282 & 0.147 & $5.4 \%$ & 0.413 & 0.456 & $36.7 \%$ \\
\hline 36 & 2 & 100 & $2 w$ & 1.0 & true & 0.502 & 0.297 & $4.4 \%$ & 0.498 & 0.496 & $34.0 \%$ \\
\hline 37 & 2 & 500 & $1 \mathrm{~d}$ & 0.3 & false & -7.039 & -1.196 & $14.9 \%$ & 0.720 & 0.555 & $22.7 \%$ \\
\hline 38 & 2 & 500 & $1 \mathrm{~d}$ & 0.3 & true & -0.197 & 0.060 & $6.3 \%$ & 0.408 & 0.454 & $21.7 \%$ \\
\hline 39 & 2 & 500 & $1 \mathrm{~d}$ & 1.0 & false & 0.186 & 0.280 & $4.4 \%$ & 0.768 & 0.609 & $26.2 \%$ \\
\hline 40 & 2 & 500 & $1 \mathrm{~d}$ & 1.0 & true & 0.510 & 0.374 & $3.9 \%$ & 0.516 & 0.469 & $37.5 \%$ \\
\hline 41 & 2 & 500 & $5 \mathrm{~d}$ & 0.3 & false & $-5 E+1$ & -3.945 & $32.8 \%$ & 0.708 & 0.582 & $31.2 \%$ \\
\hline 42 & 2 & 500 & $5 \mathrm{~d}$ & 0.3 & true & 0.291 & 0.210 & $5.0 \%$ & 0.596 & 0.547 & $29.9 \%$ \\
\hline 43 & 2 & 500 & $5 \mathrm{~d}$ & 1.0 & false & 0.244 & 0.261 & $4.6 \%$ & 0.806 & 0.681 & $21.1 \%$ \\
\hline 44 & 2 & 500 & $5 \mathrm{~d}$ & 1.0 & true & 0.186 & 0.252 & $4.6 \%$ & 0.796 & 0.675 & $20.9 \%$ \\
\hline 45 & 2 & 500 & $2 w$ & 0.3 & false & 0.383 & 0.235 & $4.8 \%$ & 0.465 & 0.476 & $35.3 \%$ \\
\hline 46 & 2 & 500 & $2 \mathrm{w}$ & 0.3 & true & 0.603 & 0.392 & $3.8 \%$ & 0.512 & 0.495 & $34.0 \%$ \\
\hline 47 & 2 & 500 & $2 w$ & 1.0 & false & 0.354 & 0.206 & $5.0 \%$ & 0.494 & 0.471 & $35.7 \%$ \\
\hline 48 & 2 & 500 & $2 w$ & 1.0 & true & 0.521 & 0.327 & $4.2 \%$ & 0.649 & 0.588 & $27.5 \%$ \\
\hline
\end{tabular}


Table S3. Parameter assignments and error metrics for all the configurations of Experiment 1 (Blue River), Scenario 3 (February $\left.2^{\text {th }}, 1998\right)$. Configuration 0 corresponds to the control open-loop model. All other errors are computed using the mean streamflow from the OPTIMISTS ensemble.

\begin{tabular}{|c|c|c|c|c|c|c|c|c|c|c|c|}
\hline \multirow{2}{*}{ Config. } & \multirow{2}{*}{ Objs. } & \multirow{2}{*}{$n$} & \multirow{2}{*}{$\Delta t$} & \multirow{2}{*}{$p_{\text {samp }}$} & \multirow{2}{*}{$k_{\text {F-class }}$} & \multicolumn{3}{|c|}{ Assimilation period } & \multicolumn{3}{|c|}{ Forecast period } \\
\hline & & & & & & $\mathrm{NSE}_{\ell 2}$ & $\mathrm{NSE}_{11}$ & MARE & $\mathrm{NSE}_{12}$ & $\mathrm{NSE}_{\ell 1}$ & MARE \\
\hline 0 & & No da & a assir & iilation & & 0.315 & 0.192 & $70.3 \%$ & $-1 \mathrm{E}+1$ & -2.519 & $40.3 \%$ \\
\hline 1 & 1 & 100 & $1 \mathrm{~d}$ & 0.3 & false & -0.218 & 0.034 & $152.0 \%$ & $-1 E+3$ & $-4 E+1$ & $427.9 \%$ \\
\hline 2 & 1 & 100 & $1 \mathrm{~d}$ & 0.3 & true & -0.464 & -0.059 & $136.6 \%$ & $-1 \mathrm{E}+1$ & -2.495 & $40.3 \%$ \\
\hline 3 & 1 & 100 & $1 \mathrm{~d}$ & 1.0 & false & 0.253 & 0.386 & $33.7 \%$ & -7.384 & -2.041 & $34.9 \%$ \\
\hline 4 & 1 & 100 & $1 \mathrm{~d}$ & 1.0 & true & 0.403 & 0.379 & $56.1 \%$ & $-4 \mathrm{E}+1$ & -5.775 & $74.5 \%$ \\
\hline 5 & 1 & 100 & $5 \mathrm{~d}$ & 0.3 & false & 0.247 & 0.433 & $25.6 \%$ & -5.781 & -0.978 & $22.9 \%$ \\
\hline 6 & 1 & 100 & $5 \mathrm{~d}$ & 0.3 & true & 0.057 & 0.432 & $17.5 \%$ & -0.319 & -0.209 & $13.6 \%$ \\
\hline 7 & 1 & 100 & $5 \mathrm{~d}$ & 1.0 & false & 0.209 & 0.411 & $29.0 \%$ & -4.083 & -1.383 & $27.3 \%$ \\
\hline 8 & 1 & 100 & $5 \mathrm{~d}$ & 1.0 & true & 0.002 & 0.421 & $16.0 \%$ & 0.302 & 0.360 & $6.8 \%$ \\
\hline 9 & 1 & 100 & $2 w$ & 0.3 & false & 0.199 & 0.390 & $33.3 \%$ & -3.279 & -1.197 & $24.9 \%$ \\
\hline 10 & 1 & 100 & $2 \mathrm{w}$ & 0.3 & true & 0.014 & 0.418 & $17.0 \%$ & -0.287 & -0.083 & $12.3 \%$ \\
\hline 11 & 1 & 100 & $2 \mathrm{w}$ & 1.0 & false & 0.239 & 0.383 & $35.4 \%$ & -5.720 & -1.754 & $31.5 \%$ \\
\hline 12 & 1 & 100 & $2 \mathrm{w}$ & 1.0 & true & -0.008 & 0.414 & $16.3 \%$ & 0.285 & 0.304 & $7.7 \%$ \\
\hline 13 & 1 & 500 & $1 \mathrm{~d}$ & 0.3 & false & -0.855 & -0.064 & $123.9 \%$ & $-1 E+1$ & -3.189 & $48.4 \%$ \\
\hline 14 & 1 & 500 & $1 \mathrm{~d}$ & 0.3 & true & $-5 E+3$ & $-7 E+1$ & $9 \mathrm{E}+1$ & $-4 \mathrm{E}+6$ & $-1 E+3$ & $1 \mathrm{E}+2$ \\
\hline 15 & 1 & 500 & $1 \mathrm{~d}$ & 1.0 & false & 0.280 & 0.395 & $33.4 \%$ & -8.686 & -2.167 & $36.8 \%$ \\
\hline 16 & 1 & 500 & $1 \mathrm{~d}$ & 1.0 & true & 0.048 & 0.431 & $15.2 \%$ & 0.035 & 0.325 & $6.8 \%$ \\
\hline 17 & 1 & 500 & $5 \mathrm{~d}$ & 0.3 & false & 0.111 & 0.455 & $17.7 \%$ & -1.759 & -0.485 & $15.7 \%$ \\
\hline 18 & 1 & 500 & $5 \mathrm{~d}$ & 0.3 & true & 0.116 & 0.442 & $19.8 \%$ & -3.878 & -1.084 & $22.9 \%$ \\
\hline 19 & 1 & 500 & $5 \mathrm{~d}$ & 1.0 & false & 0.214 & 0.420 & $27.6 \%$ & -4.184 & -1.443 & $27.9 \%$ \\
\hline 20 & 1 & 500 & $5 \mathrm{~d}$ & 1.0 & true & -0.010 & 0.421 & $15.2 \%$ & -0.275 & 0.098 & $9.3 \%$ \\
\hline 21 & 1 & 500 & $2 \mathrm{w}$ & 0.3 & false & 0.123 & 0.457 & $16.7 \%$ & -1.284 & -0.222 & $13.4 \%$ \\
\hline 22 & 1 & 500 & $2 \mathrm{w}$ & 0.3 & true & 0.000 & 0.420 & $15.7 \%$ & -0.327 & 0.088 & $10.3 \%$ \\
\hline 23 & 1 & 500 & $2 \mathrm{w}$ & 1.0 & false & 0.206 & 0.392 & $32.8 \%$ & -4.693 & -1.571 & $29.2 \%$ \\
\hline 24 & 1 & 500 & $2 \mathrm{w}$ & 1.0 & true & -0.011 & 0.413 & $16.4 \%$ & 0.385 & 0.467 & $5.5 \%$ \\
\hline 25 & 2 & 100 & $1 \mathrm{~d}$ & 0.3 & false & -0.156 & 0.179 & $74.0 \%$ & $-6 E+1$ & -6.952 & $90.9 \%$ \\
\hline 26 & 2 & 100 & $1 \mathrm{~d}$ & 0.3 & true & 0.014 & 0.292 & $50.7 \%$ & -1.904 & -0.272 & $14.6 \%$ \\
\hline 27 & 2 & 100 & $1 \mathrm{~d}$ & 1.0 & false & 0.260 & 0.407 & $30.7 \%$ & -5.529 & -1.672 & $30.7 \%$ \\
\hline 28 & 2 & 100 & $1 \mathrm{~d}$ & 1.0 & true & 0.102 & 0.380 & $29.4 \%$ & -1.515 & -0.509 & $15.9 \%$ \\
\hline 29 & 2 & 100 & $5 \mathrm{~d}$ & 0.3 & false & 0.218 & 0.406 & $30.5 \%$ & -2.345 & -0.915 & $21.8 \%$ \\
\hline 30 & 2 & 100 & $5 \mathrm{~d}$ & 0.3 & true & 0.033 & 0.404 & $21.3 \%$ & -0.605 & -0.013 & $11.6 \%$ \\
\hline 31 & 2 & 100 & $5 \mathrm{~d}$ & 1.0 & false & 0.239 & 0.398 & $32.4 \%$ & -5.079 & -1.642 & $30.2 \%$ \\
\hline 32 & 2 & 100 & $5 \mathrm{~d}$ & 1.0 & true & 0.002 & 0.403 & $19.4 \%$ & -0.370 & 0.002 & $11.3 \%$ \\
\hline 33 & 2 & 100 & $2 \mathrm{w}$ & 0.3 & false & 0.268 & 0.377 & $37.1 \%$ & -5.231 & -1.635 & $30.2 \%$ \\
\hline 34 & 2 & 100 & $2 \mathrm{w}$ & 0.3 & true & 0.050 & 0.431 & $17.2 \%$ & 0.055 & 0.312 & $7.5 \%$ \\
\hline 35 & 2 & 100 & $2 w$ & 1.0 & false & 0.253 & 0.364 & $39.3 \%$ & -6.679 & -1.948 & $33.6 \%$ \\
\hline 36 & 2 & 100 & $2 \mathrm{w}$ & 1.0 & true & 0.053 & 0.425 & $19.2 \%$ & -0.709 & -0.255 & $14.4 \%$ \\
\hline 37 & 2 & 500 & $1 \mathrm{~d}$ & 0.3 & false & -0.127 & 0.185 & $72.1 \%$ & -1.810 & -0.453 & $15.4 \%$ \\
\hline 38 & 2 & 500 & $1 \mathrm{~d}$ & 0.3 & true & -0.221 & 0.179 & $120.8 \%$ & $-3 E+2$ & $-2 E+1$ & $197.3 \%$ \\
\hline 39 & 2 & 500 & $1 \mathrm{~d}$ & 1.0 & false & 0.270 & 0.414 & $29.5 \%$ & -6.369 & -1.731 & $31.8 \%$ \\
\hline
\end{tabular}




\begin{tabular}{|c|c|c|c|c|c|c|c|c|c|c|c|}
\hline \multirow{2}{*}{ Config. } & \multirow{2}{*}{ Objs. } & \multirow{2}{*}{$n$} & \multirow{2}{*}{$\Delta t$} & \multirow{2}{*}{$p_{\text {samp }}$} & \multirow{2}{*}{$k_{\text {F-class }}$} & \multicolumn{3}{|c|}{ Assimilation period } & \multicolumn{3}{|c|}{ Forecast period } \\
\hline & & & & & & $\mathrm{NSE}_{\ell 2}$ & NSE $_{\ell 1}$ & MARE & $\mathrm{NSE}_{12}$ & $\mathrm{NSE}_{\ell 1}$ & MARE \\
\hline 40 & 2 & 500 & $1 \mathrm{~d}$ & 1.0 & true & 0.021 & 0.427 & $15.5 \%$ & -0.433 & 0.036 & $10.0 \%$ \\
\hline 41 & 2 & 500 & $5 \mathrm{~d}$ & 0.3 & false & 0.195 & 0.451 & $22.0 \%$ & -0.667 & -0.125 & $12.6 \%$ \\
\hline 42 & 2 & 500 & $5 \mathrm{~d}$ & 0.3 & true & 0.088 & 0.444 & $18.3 \%$ & -0.992 & 0.045 & $10.9 \%$ \\
\hline 43 & 2 & 500 & $5 \mathrm{~d}$ & 1.0 & false & 0.197 & 0.407 & $29.8 \%$ & -2.852 & -1.116 & $23.9 \%$ \\
\hline 44 & 2 & 500 & $5 \mathrm{~d}$ & 1.0 & true & 0.043 & 0.432 & $16.8 \%$ & 0.354 & 0.344 & $7.1 \%$ \\
\hline 45 & 2 & 500 & $2 \mathrm{w}$ & 0.3 & false & 0.182 & 0.456 & $20.6 \%$ & -0.224 & -0.105 & $12.0 \%$ \\
\hline 46 & 2 & 500 & $2 \mathrm{w}$ & 0.3 & true & 0.001 & 0.423 & $15.6 \%$ & 0.105 & 0.270 & $8.1 \%$ \\
\hline 47 & 2 & 500 & $2 \mathrm{w}$ & 1.0 & false & 0.245 & 0.379 & $36.1 \%$ & -5.616 & -1.747 & $31.4 \%$ \\
\hline 48 & 2 & 500 & $2 \mathrm{w}$ & 1.0 & true & 0.034 & 0.431 & $16.5 \%$ & 0.230 & 0.188 & $9.1 \%$ \\
\hline
\end{tabular}


Table S4. Parameter assignments and error metrics for all the configurations of Experiment 2 (Indiantown Run), Scenario 1 (July $\left.26^{\text {th }}, 2009\right)$. Configuration 0 corresponds to the control open-loop model. All other errors are computed using the mean streamflow from the OPTIMISTS ensemble.

\begin{tabular}{|c|c|c|c|c|c|c|c|c|c|c|c|}
\hline \multirow{2}{*}{ Config. } & \multirow{2}{*}{$\Delta t$} & \multirow{2}{*}{ Objs. } & \multirow{2}{*}{$n$} & \multirow{2}{*}{$p_{\text {samp }}$} & \multirow{2}{*}{$w_{\text {root }}$} & \multicolumn{3}{|c|}{ Assimilation period } & \multicolumn{3}{|c|}{ Forecast period } \\
\hline & & & & & & $\mathrm{NSE}_{12}$ & $\mathrm{NSE}_{t 1}$ & MARE & $\mathrm{NSE}_{12}$ & $\mathrm{NSE}_{\ell 1}$ & MARE \\
\hline 0 & & - No dat & assin & ilation & & 0.478 & -0.015 & $24.9 \%$ & 0.022 & -0.003 & $54.3 \%$ \\
\hline 1 & $1 \mathrm{~h}$ & 2 & 100 & 0.25 & $60 \%$ & $-9 E+04$ & $-1 \mathrm{E}+02$ & $2.8 \mathrm{E}+01$ & 0.067 & -0.759 & $197.1 \%$ \\
\hline 2 & $1 \mathrm{~h}$ & 2 & 100 & 0.25 & $95 \%$ & $-2 \mathrm{E}+03$ & $-3 E+01$ & $7.4 \mathrm{E}+00$ & 0.365 & 0.193 & $73.5 \%$ \\
\hline 3 & $1 \mathrm{~h}$ & 2 & 100 & 1.00 & $60 \%$ & $-1 \mathrm{E}+01$ & -3.324 & $98.3 \%$ & 0.279 & -0.360 & $143.9 \%$ \\
\hline 4 & $1 \mathrm{~h}$ & 2 & 100 & 1.00 & $95 \%$ & -1.455 & -0.862 & $44.9 \%$ & 0.346 & -0.099 & $107.0 \%$ \\
\hline 5 & $1 \mathrm{~h}$ & 2 & 200 & 0.25 & $60 \%$ & $-2 \mathrm{E}+05$ & $-1 E+02$ & $3.4 \mathrm{E}+01$ & 0.075 & -0.769 & $196.1 \%$ \\
\hline 6 & $1 \mathrm{~h}$ & 2 & 200 & 0.25 & $95 \%$ & $-3 E+03$ & $-3 \mathrm{E}+01$ & $6.8 \mathrm{E}+00$ & 0.357 & 0.275 & $62.2 \%$ \\
\hline 7 & $1 \mathrm{~h}$ & 2 & 200 & 1.00 & $60 \%$ & $-1 \mathrm{E}+01$ & -3.900 & $111.6 \%$ & 0.284 & -0.330 & $143.5 \%$ \\
\hline 8 & $1 \mathrm{~h}$ & 2 & 200 & 1.00 & $95 \%$ & -2.320 & -1.192 & $54.1 \%$ & 0.341 & -0.182 & $120.1 \%$ \\
\hline 9 & $2 w$ & 2 & 100 & 0.25 & $60 \%$ & 0.471 & 0.208 & $18.1 \%$ & 0.317 & 0.381 & $43.2 \%$ \\
\hline 10 & $2 \mathrm{w}$ & 2 & 100 & 0.25 & $95 \%$ & 0.099 & -0.001 & $23.2 \%$ & 0.320 & 0.375 & $44.3 \%$ \\
\hline 11 & $2 w$ & 2 & 100 & 1.00 & $60 \%$ & 0.571 & 0.371 & $13.3 \%$ & 0.292 & 0.393 & $38.3 \%$ \\
\hline 12 & $2 w$ & 2 & 100 & 1.00 & $95 \%$ & 0.572 & 0.353 & $14.1 \%$ & 0.323 & 0.373 & $45.7 \%$ \\
\hline 13 & $2 w$ & 2 & 200 & 0.25 & $60 \%$ & 0.531 & 0.295 & $15.5 \%$ & 0.327 & 0.367 & $47.0 \%$ \\
\hline 14 & $2 w$ & 2 & 200 & 0.25 & $95 \%$ & 0.520 & 0.305 & $15.4 \%$ & 0.312 & 0.380 & $42.6 \%$ \\
\hline 15 & $2 \mathrm{w}$ & 2 & 200 & 1.00 & $60 \%$ & 0.360 & 0.211 & $17.5 \%$ & 0.322 & 0.338 & $50.1 \%$ \\
\hline 16 & $2 w$ & 2 & 200 & 1.00 & $95 \%$ & 0.427 & 0.175 & $18.7 \%$ & 0.332 & 0.357 & $49.0 \%$ \\
\hline 17 & $1 \mathrm{~h}$ & 1 & 100 & 0.25 & $60 \%$ & $-5 E+02$ & $-3 E+01$ & $7.0 \mathrm{E}+00$ & -0.429 & -1.338 & $2.8 \mathrm{E}+00$ \\
\hline 18 & $1 \mathrm{~h}$ & 1 & 100 & 0.25 & $95 \%$ & $-5 E+01$ & -7.655 & $196.9 \%$ & 0.094 & -0.736 & $193.1 \%$ \\
\hline 19 & $1 \mathrm{~h}$ & 1 & 100 & 1.00 & $60 \%$ & $-1 E+01$ & -3.201 & $96.0 \%$ & 0.314 & -0.269 & $132.4 \%$ \\
\hline 20 & $1 \mathrm{~h}$ & 1 & 100 & 1.00 & $95 \%$ & -9.818 & -3.016 & $90.8 \%$ & 0.290 & -0.354 & $142.2 \%$ \\
\hline 21 & $1 \mathrm{~h}$ & 1 & 200 & 0.25 & $60 \%$ & $-2 E+05$ & $-6 E+02$ & $1.4 \mathrm{E}+02$ & $-5 E+01$ & -7.699 & $1.4 \mathrm{E}+01$ \\
\hline 22 & $1 \mathrm{~h}$ & 1 & 200 & 0.25 & $95 \%$ & $-1 E+05$ & $-4 \mathrm{E}+02$ & $9.7 \mathrm{E}+01$ & $-5 E+01$ & -7.337 & $1.4 \mathrm{E}+01$ \\
\hline 23 & $1 \mathrm{~h}$ & 1 & 200 & 1.00 & $60 \%$ & $-1 E+01$ & -3.965 & $113.2 \%$ & 0.254 & -0.447 & $153.8 \%$ \\
\hline 24 & $1 \mathrm{~h}$ & 1 & 200 & 1.00 & $95 \%$ & $-1 E+01$ & -3.601 & $103.3 \%$ & 0.283 & -0.374 & $144.9 \%$ \\
\hline 25 & $2 w$ & 1 & 100 & 0.25 & $60 \%$ & 0.453 & 0.071 & $21.8 \%$ & 0.320 & 0.373 & $44.2 \%$ \\
\hline 26 & $2 w$ & 1 & 100 & 0.25 & $95 \%$ & 0.279 & -0.053 & $24.6 \%$ & 0.329 & 0.368 & $46.3 \%$ \\
\hline 27 & $2 w$ & 1 & 100 & 1.00 & $60 \%$ & 0.500 & 0.177 & $18.9 \%$ & 0.331 & 0.369 & $46.3 \%$ \\
\hline 28 & $2 \mathrm{w}$ & 1 & 100 & 1.00 & $95 \%$ & 0.358 & 0.041 & $22.2 \%$ & 0.330 & 0.364 & $47.4 \%$ \\
\hline 29 & $2 w$ & 1 & 200 & 0.25 & $60 \%$ & 0.494 & 0.177 & $18.8 \%$ & 0.324 & 0.366 & $46.3 \%$ \\
\hline 30 & $2 w$ & 1 & 200 & 0.25 & $95 \%$ & 0.362 & 0.036 & $22.3 \%$ & 0.329 & 0.362 & $47.4 \%$ \\
\hline 31 & $2 w$ & 1 & 200 & 1.00 & $60 \%$ & 0.452 & 0.158 & $19.2 \%$ & 0.329 & 0.362 & $46.7 \%$ \\
\hline 32 & $2 \mathrm{w}$ & 1 & 200 & 1.00 & $95 \%$ & 0.480 & 0.155 & $19.4 \%$ & 0.333 & 0.362 & $47.2 \%$ \\
\hline
\end{tabular}


Table S5. Parameter assignments and error metrics for all the configurations of Experiment 2 (Indiantown Run), Scenario 2 (August $\mathbf{2 6}^{\text {th }}, 2009$ ). Configuration 0 corresponds to the control open-loop model. All other errors are computed using the mean streamflow from the OPTIMISTS ensemble.

\begin{tabular}{|c|c|c|c|c|c|c|c|c|c|c|c|}
\hline \multirow{2}{*}{ Config. } & \multirow{2}{*}{$\Delta t$} & \multirow{2}{*}{ Objs. } & \multirow{2}{*}{$n$} & \multirow{2}{*}{$p_{\text {samp }}$} & \multirow{2}{*}{$w_{\text {root }}$} & \multicolumn{3}{|c|}{ Assimilation period } & \multicolumn{3}{|c|}{ Forecast period } \\
\hline & & & & & & $\mathrm{NSE}_{12}$ & $\mathrm{NSE}_{\ell 1}$ & MARE & $\mathrm{NSE}_{12}$ & $\mathrm{NSE}_{\ell 1}$ & MARE \\
\hline 0 & & - No dat & assim & ilation & & -0.926 & -0.963 & $68.0 \%$ & -0.428 & -0.733 & $58.3 \%$ \\
\hline 1 & $1 \mathrm{~h}$ & 2 & 100 & 0.25 & $60 \%$ & $-8 \mathrm{E}+02$ & $-2 \mathrm{E}+01$ & $8.5 \mathrm{E}+00$ & -5.171 & -2.290 & $104.3 \%$ \\
\hline 2 & $1 \mathrm{~h}$ & 2 & 100 & 0.25 & $95 \%$ & $-2 \mathrm{E}+02$ & -7.769 & $3.9 \mathrm{E}+00$ & -0.581 & -0.517 & $46.2 \%$ \\
\hline 3 & $1 \mathrm{~h}$ & 2 & 100 & 1.00 & $60 \%$ & 0.672 & 0.570 & $16.1 \%$ & -1.459 & -1.065 & $66.7 \%$ \\
\hline 4 & $1 \mathrm{~h}$ & 2 & 100 & 1.00 & $95 \%$ & 0.637 & 0.643 & $11.4 \%$ & -0.971 & -0.799 & $57.0 \%$ \\
\hline 5 & $1 \mathrm{~h}$ & 2 & 200 & 0.25 & $60 \%$ & $-5 E+03$ & $-3 \mathrm{E}+01$ & $1.0 \mathrm{E}+01$ & $-1 E+01$ & -3.291 & $134.0 \%$ \\
\hline 6 & $1 \mathrm{~h}$ & 2 & 200 & 0.25 & $95 \%$ & $-3 E+02$ & -9.816 & $5.0 \mathrm{E}+00$ & -1.156 & -0.797 & $54.9 \%$ \\
\hline 7 & $1 \mathrm{~h}$ & 2 & 200 & 1.00 & $60 \%$ & 0.708 & 0.518 & $19.2 \%$ & -1.493 & -1.082 & $67.2 \%$ \\
\hline 8 & $1 \mathrm{~h}$ & 2 & 200 & 1.00 & $95 \%$ & 0.657 & 0.625 & $12.9 \%$ & -1.259 & -0.966 & $63.0 \%$ \\
\hline 9 & $2 w$ & 2 & 100 & 0.25 & $60 \%$ & -0.607 & -0.154 & $35.9 \%$ & 0.322 & 0.118 & $25.8 \%$ \\
\hline 10 & $2 w$ & 2 & 100 & 0.25 & $95 \%$ & 0.041 & 0.013 & $29.1 \%$ & 0.248 & 0.070 & $27.1 \%$ \\
\hline 11 & $2 w$ & 2 & 100 & 1.00 & $60 \%$ & 0.122 & 0.096 & $25.6 \%$ & 0.048 & -0.067 & $31.1 \%$ \\
\hline 12 & $2 w$ & 2 & 100 & 1.00 & $95 \%$ & -0.113 & -0.164 & $35.1 \%$ & 0.451 & 0.212 & $23.2 \%$ \\
\hline 13 & $2 w$ & 2 & 200 & 0.25 & $60 \%$ & 0.186 & 0.161 & $23.5 \%$ & 0.051 & -0.089 & $32.1 \%$ \\
\hline 14 & $2 w$ & 2 & 200 & 0.25 & $95 \%$ & 0.118 & 0.088 & $26.0 \%$ & 0.132 & -0.010 & $29.4 \%$ \\
\hline 15 & $2 w$ & 2 & 200 & 1.00 & $60 \%$ & -0.019 & -0.052 & $30.9 \%$ & 0.255 & 0.077 & $26.9 \%$ \\
\hline 16 & $2 w$ & 2 & 200 & 1.00 & $95 \%$ & 0.022 & -0.030 & $30.3 \%$ & 0.279 & 0.085 & $26.8 \%$ \\
\hline 17 & $1 \mathrm{~h}$ & 1 & 100 & 0.25 & $60 \%$ & -3.015 & -2.226 & $138.7 \%$ & $-2 \mathrm{E}+01$ & -4.216 & $164.2 \%$ \\
\hline 18 & $1 \mathrm{~h}$ & 1 & 100 & 0.25 & $95 \%$ & -0.028 & -0.550 & $61.0 \%$ & -7.080 & -2.694 & $115.6 \%$ \\
\hline 19 & $1 \mathrm{~h}$ & 1 & 100 & 1.00 & $60 \%$ & 0.539 & 0.542 & $14.8 \%$ & -1.544 & -1.083 & $66.8 \%$ \\
\hline 20 & $1 \mathrm{~h}$ & 1 & 100 & 1.00 & $95 \%$ & 0.565 & 0.442 & $21.7 \%$ & -2.307 & -1.433 & $78.4 \%$ \\
\hline 21 & $1 \mathrm{~h}$ & 1 & 200 & 0.25 & $60 \%$ & $-5 E+03$ & $-1 \mathrm{E}+02$ & $4.6 \mathrm{E}+01$ & $-6 \mathrm{E}+02$ & $-2 \mathrm{E}+01$ & $8.5 \mathrm{E}+00$ \\
\hline 22 & $1 \mathrm{~h}$ & 1 & 200 & 0.25 & $95 \%$ & $-1 E+04$ & $-1 \mathrm{E}+02$ & $4.9 \mathrm{E}+01$ & $-7 E+02$ & $-2 \mathrm{E}+01$ & $9.4 \mathrm{E}+00$ \\
\hline 23 & $1 \mathrm{~h}$ & 1 & 200 & 1.00 & $60 \%$ & 0.538 & 0.548 & $16.1 \%$ & -2.240 & -1.396 & $77.8 \%$ \\
\hline 24 & $1 \mathrm{~h}$ & 1 & 200 & 1.00 & $95 \%$ & 0.551 & 0.594 & $12.7 \%$ & -2.089 & -1.318 & $74.2 \%$ \\
\hline 25 & $2 \mathrm{w}$ & 1 & 100 & 0.25 & $60 \%$ & 0.272 & 0.414 & $15.6 \%$ & -0.859 & -0.577 & $46.7 \%$ \\
\hline 26 & $2 \mathrm{w}$ & 1 & 100 & 0.25 & $95 \%$ & 0.307 & 0.422 & $14.7 \%$ & -0.779 & -0.494 & $43.5 \%$ \\
\hline 27 & $2 \mathrm{w}$ & 1 & 100 & 1.00 & $60 \%$ & 0.230 & 0.300 & $18.2 \%$ & -0.501 & -0.368 & $39.8 \%$ \\
\hline 28 & $2 \mathrm{w}$ & 1 & 100 & 1.00 & $95 \%$ & 0.220 & 0.268 & $19.4 \%$ & -0.475 & -0.359 & $39.6 \%$ \\
\hline 29 & $2 w$ & 1 & 200 & 0.25 & $60 \%$ & 0.296 & 0.460 & $14.6 \%$ & -0.757 & -0.527 & $45.2 \%$ \\
\hline 30 & $2 \mathrm{w}$ & 1 & 200 & 0.25 & $95 \%$ & 0.377 & 0.485 & $13.8 \%$ & -0.916 & -0.564 & $45.7 \%$ \\
\hline 31 & $2 \mathrm{w}$ & 1 & 200 & 1.00 & $60 \%$ & 0.207 & 0.285 & $18.8 \%$ & -0.493 & -0.348 & $39.0 \%$ \\
\hline 32 & $2 \mathrm{w}$ & 1 & 200 & 1.00 & $95 \%$ & 0.202 & 0.278 & $18.9 \%$ & -0.525 & -0.382 & $40.3 \%$ \\
\hline
\end{tabular}


Table S6. Parameter assignments and error metrics for all the configurations of Experiment 3 (Indiantown Run), Scenario 1 (July $26^{\text {th }}, 2009$ ). Configuration 0 corresponds to the control open-loop model. All other errors are computed using the mean streamflow from the OPTIMISTS ensemble.

\begin{tabular}{|c|c|c|c|c|c|c|c|c|c|}
\hline \multirow{2}{*}{ Config. } & \multirow{2}{*}{$\Delta t$} & \multirow{2}{*}{$p_{\text {samp }}$} & \multirow{2}{*}{$g$} & \multicolumn{3}{|c|}{ Assimilation period } & \multicolumn{3}{|c|}{ Forecast period } \\
\hline & & & & $\mathrm{NSE}_{\ell 2}$ & $\mathrm{NSE}_{\ell 1}$ & MARE & $\mathrm{NSE}_{\ell 2}$ & $\mathrm{NSE}_{\ell 1}$ & MARE \\
\hline 0 & -- No data & ssimila & n -- & 0.478 & -0.015 & $24.9 \%$ & 0.022 & -0.003 & $54.3 \%$ \\
\hline 1 & $1 \mathrm{~h}$ & 0.4 & 0.5 & $-1 \mathrm{E}+05$ & $-2 \mathrm{E}+02$ & $5.2 \mathrm{E}+01$ & 0.359 & 0.286 & $60.6 \%$ \\
\hline 2 & $1 \mathrm{~h}$ & 0.4 & 1.0 & $-5 \mathrm{E}+03$ & $-3 \mathrm{E}+01$ & $8.3 \mathrm{E}+00$ & 0.366 & 0.271 & $63.1 \%$ \\
\hline 3 & $1 \mathrm{~h}$ & 1.0 & 0.5 & -3.682 & -1.729 & $64.1 \%$ & 0.358 & -0.069 & $107.8 \%$ \\
\hline 4 & $1 \mathrm{~h}$ & 1.0 & 1.0 & -2.038 & -1.146 & $50.9 \%$ & 0.371 & 0.031 & $94.3 \%$ \\
\hline 5 & $6 \mathrm{~h}$ & 0.4 & 0.5 & $-6 E+02$ & $-1 \mathrm{E}+01$ & $2.9 \mathrm{E}+00$ & 0.354 & 0.307 & $57.1 \%$ \\
\hline 6 & $6 \mathrm{~h}$ & 0.4 & 1.0 & $-9 \mathrm{E}+02$ & $-1 \mathrm{E}+01$ & $317.4 \%$ & 0.357 & 0.291 & $59.9 \%$ \\
\hline 7 & $6 \mathrm{~h}$ & 1.0 & 0.5 & -2.107 & -1.230 & $52.0 \%$ & 0.357 & 0.086 & $84.0 \%$ \\
\hline 8 & $6 \mathrm{~h}$ & 1.0 & 1.0 & -1.431 & -0.889 & $43.0 \%$ & 0.361 & 0.292 & $60.1 \%$ \\
\hline 9 & $1 \mathrm{~d}$ & 0.4 & 0.5 & $-2 \mathrm{E}+02$ & -4.030 & $119.4 \%$ & 0.351 & 0.312 & $56.1 \%$ \\
\hline 10 & $1 \mathrm{~d}$ & 0.4 & 1.0 & $-2 \mathrm{E}+03$ & -6.205 & $171.3 \%$ & 0.350 & 0.340 & $52.3 \%$ \\
\hline 11 & $1 \mathrm{~d}$ & 1.0 & 0.5 & -1.406 & -0.891 & $42.8 \%$ & 0.353 & 0.325 & $55.0 \%$ \\
\hline 12 & $1 \mathrm{~d}$ & 1.0 & 1.0 & -1.440 & -0.893 & $42.9 \%$ & 0.352 & 0.315 & $56.6 \%$ \\
\hline 13 & $3.5 \mathrm{~d}$ & 0.4 & 0.5 & $-3 E+03$ & -7.748 & $2.1 \mathrm{E}+00$ & 0.337 & 0.311 & $54.2 \%$ \\
\hline 14 & $3.5 \mathrm{~d}$ & 0.4 & 1.0 & $-6 \mathrm{E}+01$ & -1.096 & $49.0 \%$ & 0.328 & 0.337 & $50.2 \%$ \\
\hline 15 & $3.5 \mathrm{~d}$ & 1.0 & 0.5 & 0.224 & 0.067 & $22.1 \%$ & 0.331 & 0.306 & $55.4 \%$ \\
\hline 16 & $3.5 \mathrm{~d}$ & 1.0 & 1.0 & 0.058 & -0.095 & $25.6 \%$ & 0.333 & 0.360 & $48.8 \%$ \\
\hline 17 & $2 w$ & 0.4 & 0.5 & $-8 \mathrm{E}+01$ & -1.527 & $59.6 \%$ & 0.339 & 0.326 & $54.1 \%$ \\
\hline 18 & $2 \mathrm{w}$ & 0.4 & 1.0 & 0.133 & -0.190 & $28.1 \%$ & 0.333 & 0.357 & $49.0 \%$ \\
\hline 19 & $2 \mathrm{w}$ & 1.0 & 0.5 & 0.399 & 0.242 & $16.3 \%$ & 0.301 & 0.394 & $40.2 \%$ \\
\hline 20 & $2 \mathrm{w}$ & 1.0 & 1.0 & 0.560 & 0.340 & $14.4 \%$ & 0.308 & 0.389 & $41.3 \%$ \\
\hline 21 & $4 \mathrm{w}$ & 0.4 & 0.5 & -0.743 & -0.735 & $42.0 \%$ & 0.355 & 0.112 & $79.6 \%$ \\
\hline 22 & $4 \mathrm{w}$ & 0.4 & 1.0 & -1.469 & -1.161 & $52.2 \%$ & 0.358 & 0.047 & $87.4 \%$ \\
\hline 23 & $4 \mathrm{w}$ & 1.0 & 0.5 & -2.873 & -1.763 & $66.4 \%$ & 0.355 & 0.033 & $90.8 \%$ \\
\hline 24 & $4 w$ & 1.0 & 1.0 & -0.577 & -0.622 & $39.2 \%$ & 0.351 & 0.159 & $74.2 \%$ \\
\hline
\end{tabular}


Table S7. Parameter assignments and error metrics for all the configurations of Experiment 3 (Indiantown Run), Scenario 2 (August $\mathbf{2 6}^{\text {th }}, 2009$ ). Configuration 0 corresponds to the control open-loop model. All other errors are computed using the mean streamflow from the OPTIMISTS ensemble.

\begin{tabular}{|c|c|c|c|c|c|c|c|c|c|}
\hline \multirow{2}{*}{ Config. } & \multirow{2}{*}{$\Delta t$} & \multirow{2}{*}{$p_{\text {samp }}$} & \multirow{2}{*}{$g$} & \multicolumn{3}{|c|}{ Assimilation period } & \multicolumn{3}{|c|}{ Forecast period } \\
\hline & & & & $\mathrm{NSE}_{\ell 2}$ & $\mathrm{NSE}_{\ell 1}$ & MARE & $\mathrm{NSE}_{\ell 2}$ & $\mathrm{NSE}_{\ell 1}$ & MARE \\
\hline 0 & -- No data & ssimila & n -- & -0.926 & -0.963 & $68.0 \%$ & -0.428 & -0.733 & $58.3 \%$ \\
\hline 1 & $1 \mathrm{~h}$ & 0.4 & 0.5 & $-1 E+03$ & $-1 \mathrm{E}+01$ & $6.2 \mathrm{E}+00$ & -0.133 & -0.204 & $37.1 \%$ \\
\hline 2 & $1 \mathrm{~h}$ & 0.4 & 1.0 & $-6 E+02$ & -8.948 & $4.4 \mathrm{E}+00$ & -1.516 & -0.967 & $60.3 \%$ \\
\hline 3 & $1 \mathrm{~h}$ & 1.0 & 0.5 & 0.604 & 0.686 & $8.0 \%$ & -0.352 & -0.459 & $46.3 \%$ \\
\hline 4 & $1 \mathrm{~h}$ & 1.0 & 1.0 & 0.664 & 0.596 & $14.0 \%$ & -1.591 & -1.133 & $68.3 \%$ \\
\hline 5 & $6 \mathrm{~h}$ & 0.4 & 0.5 & $-1 \mathrm{E}+02$ & -4.849 & $2.7 \mathrm{E}+00$ & -1.270 & -0.769 & $52.7 \%$ \\
\hline 6 & $6 \mathrm{~h}$ & 0.4 & 1.0 & $-2 \mathrm{E}+01$ & -0.391 & $57.4 \%$ & -0.371 & -0.393 & $42.1 \%$ \\
\hline 7 & $6 \mathrm{~h}$ & 1.0 & 0.5 & 0.529 & 0.634 & $8.6 \%$ & -1.098 & -0.679 & $49.6 \%$ \\
\hline 8 & $6 \mathrm{~h}$ & 1.0 & 1.0 & 0.494 & 0.606 & $9.2 \%$ & -0.388 & -0.460 & $45.5 \%$ \\
\hline 9 & $1 \mathrm{~d}$ & 0.4 & 0.5 & -7.399 & 0.020 & $39.8 \%$ & -0.830 & -0.563 & $46.2 \%$ \\
\hline 10 & $1 \mathrm{~d}$ & 0.4 & 1.0 & 0.475 & 0.444 & $14.3 \%$ & -0.213 & -0.286 & $38.6 \%$ \\
\hline 11 & $1 \mathrm{~d}$ & 1.0 & 0.5 & 0.398 & 0.432 & $14.7 \%$ & -1.096 & -0.717 & $51.5 \%$ \\
\hline 12 & $1 \mathrm{~d}$ & 1.0 & 1.0 & 0.453 & 0.512 & $11.9 \%$ & -0.656 & -0.501 & $44.9 \%$ \\
\hline 13 & $3.5 \mathrm{~d}$ & 0.4 & 0.5 & -1.056 & 0.047 & $27.5 \%$ & -0.706 & -0.465 & $42.7 \%$ \\
\hline 14 & $3.5 \mathrm{~d}$ & 0.4 & 1.0 & 0.296 & 0.366 & $15.6 \%$ & -0.690 & -0.437 & $41.6 \%$ \\
\hline 15 & $3.5 \mathrm{~d}$ & 1.0 & 0.5 & 0.028 & 0.076 & $24.9 \%$ & -0.454 & -0.402 & $41.9 \%$ \\
\hline 16 & $3.5 \mathrm{~d}$ & 1.0 & 1.0 & 0.122 & 0.149 & $22.7 \%$ & -0.522 & -0.376 & $40.0 \%$ \\
\hline 17 & $2 \mathrm{w}$ & 0.4 & 0.5 & $-2 E+01$ & -0.396 & $50.0 \%$ & 0.094 & -0.044 & $30.5 \%$ \\
\hline 18 & $2 w$ & 0.4 & 1.0 & -0.002 & -0.062 & $31.7 \%$ & 0.358 & 0.140 & $25.3 \%$ \\
\hline 19 & $2 \mathrm{w}$ & 1.0 & 0.5 & -0.157 & -0.171 & $35.0 \%$ & 0.340 & 0.108 & $26.4 \%$ \\
\hline 20 & $2 w$ & 1.0 & 1.0 & 0.032 & -0.024 & $30.2 \%$ & 0.301 & 0.109 & $26.0 \%$ \\
\hline 21 & $4 w$ & 0.4 & 0.5 & 0.379 & 0.491 & $11.4 \%$ & -1.398 & -0.877 & $56.6 \%$ \\
\hline 22 & $4 \mathrm{w}$ & 0.4 & 1.0 & 0.330 & 0.424 & $13.4 \%$ & -1.281 & -0.834 & $55.4 \%$ \\
\hline 23 & $4 w$ & 1.0 & 0.5 & 0.308 & 0.397 & $14.1 \%$ & -0.907 & -0.653 & $49.8 \%$ \\
\hline 24 & $4 w$ & 1.0 & 1.0 & 0.327 & 0.425 & $13.2 \%$ & -1.159 & -0.771 & $53.4 \%$ \\
\hline
\end{tabular}


Table S8. ANOVA table for Experiment 1 for the improvements in MARE with respect to the default model. DF: degrees of freedom; Adj SS: adjusted sum of squares; Adj MS: adjusted mean sum of squares; Sims.: $n$; Optimjzatijon:-pernels: $k_{F-c l a s s ~}$.

\begin{tabular}{|c|c|c|c|c|c|}
\hline Source & DF & Adj SS & Adj MS & F-Value & P-Value \\
\hline Model & 49 & 8.093 & 0.165 & 4.610 & 0.000 \\
\hline Scenario & 2 & 1.046 & 0.523 & 14.610 & 0.000 \\
\hline Linear & 6 & 3.240 & 0.540 & 15.080 & 0.000 \\
\hline Objs. & 1 & 0.275 & 0.274 & 7.660 & 0.007 \\
\hline Sims. & 1 & 0.005 & 0.005 & 0.140 & 0.704 \\
\hline TimeStep & 2 & 2.499 & 1.249 & 34.890 & 0.000 \\
\hline Optimization & 1 & 0.460 & 0.460 & 12.860 & 0.001 \\
\hline F-kernels & 1 & 0.001 & 0.001 & 0.040 & 0.850 \\
\hline 2-Way Interactions & 14 & 2.292 & 0.164 & 4.570 & 0.000 \\
\hline Objs. *Sims. & 1 & 0.000 & 0.000 & 0.000 & 0.961 \\
\hline Objs.*TimeStep & 2 & 0.507 & 0.253 & 7.080 & 0.001 \\
\hline Objs.*Optimization & 1 & 0.119 & 0.119 & 3.330 & 0.071 \\
\hline Objs.*F-kernels & 1 & 0.011 & 0.010 & 0.290 & 0.590 \\
\hline Sims.*TimeStep & 2 & 0.025 & 0.013 & 0.350 & 0.704 \\
\hline Sims.*Optimization & 1 & 0.019 & 0.019 & 0.540 & 0.464 \\
\hline Sims.*F-kernels & 1 & 0.157 & 0.157 & 4.390 & 0.039 \\
\hline TimeStep*Optimization & 2 & 1.172 & 0.586 & 16.370 & 0.000 \\
\hline TimeStep*F-kernels & 2 & 0.106 & 0.053 & 1.470 & 0.234 \\
\hline Optimization*F-kernels & 1 & 0.176 & 0.176 & 4.910 & 0.029 \\
\hline 3-Way Interactions & 16 & 1.001 & 0.063 & 1.750 & 0.051 \\
\hline Objs.*Sims.*TimeStep & 2 & 0.005 & 0.002 & 0.070 & 0.935 \\
\hline Objs. *Sims. ${ }^{*}$ Optimization & 1 & 0.000 & 0.000 & 0.010 & 0.918 \\
\hline Objs. ${ }^{*}$ Sims. ${ }^{*}$ F-kernels & 1 & 0.000 & 0.000 & 0.010 & 0.923 \\
\hline Objs.*TimeStep*Optimization & 2 & 0.233 & 0.116 & 3.250 & 0.043 \\
\hline Objs. ${ }^{*}$ TimeStep*F-kernels & 2 & 0.001 & 0.001 & 0.020 & 0.985 \\
\hline Objs. ${ }^{*}$ Optimization $*$ F-kernels & 1 & 0.012 & 0.012 & 0.320 & 0.572 \\
\hline Sims.*TimeStep*Optimization & 2 & 0.082 & 0.041 & 1.150 & 0.321 \\
\hline Sims.*TimeStep*F-kernels & 2 & 0.291 & 0.146 & 4.070 & 0.020 \\
\hline Sims.*Optimization*F-kernels & 1 & 0.227 & 0.227 & 6.340 & 0.013 \\
\hline TimeStep*Optimization*F-kernels & 2 & 0.149 & 0.074 & 2.070 & 0.131 \\
\hline 4-Way Interactions & 9 & 0.439 & 0.049 & 1.360 & 0.216 \\
\hline Objs.*Sims.*TimeStep*Optimization & 2 & 0.007 & 0.004 & 0.100 & 0.903 \\
\hline Objs. *Sims.*TimeStep*F-kernels & 2 & 0.002 & 0.001 & 0.020 & 0.978 \\
\hline Objs. $*$ Sims. $*$ Optimization*F-kernels & 1 & 0.037 & 0.037 & 1.020 & 0.315 \\
\hline Objs.*TimeStep*Optimization*F-kernels & 2 & 0.010 & 0.005 & 0.130 & 0.874 \\
\hline Sims.*TimeStep*Optimization*F-kernels & 2 & 0.384 & 0.192 & 5.370 & 0.006 \\
\hline 5-Way Interaction & 2 & 0.075 & 0.038 & 1.050 & 0.354 \\
\hline Objs. *Sims. ${ }^{*}$ TimeStep*Optimization*F-kernels & 2 & 0.075 & 0.038 & 1.050 & 0.354 \\
\hline Error & 94 & 3.366 & 0.036 & & \\
\hline Total & 143 & 11.460 & & & \\
\hline
\end{tabular}


Table S9. ANOVA table for Experiment 2 for the improvements in NSE 2 with respect to the default model. DF: degrees of freedom; Adj SS: adjusted sum of squares; Adj MS: adjusted mean sum of squares; Candidates: $n$; Roots: $w_{\text {root• }}$.

\begin{tabular}{|c|c|c|c|c|c|}
\hline Source & DF & Adj SS & Adj MS & F-Value & P-Value \\
\hline Model & 32 & 65.769 & 2.055 & 3.450 & 0.000 \\
\hline Scenario & 1 & 11.325 & 11.325 & 19.020 & 0.000 \\
\hline Linear & 5 & 40.151 & 8.030 & 13.490 & 0.000 \\
\hline Time_step & 1 & 25.442 & 25.442 & 42.730 & 0.000 \\
\hline Objectives & 1 & 7.834 & 7.834 & 13.160 & 0.001 \\
\hline Candidates & 1 & 0.866 & 0.866 & 1.450 & 0.237 \\
\hline Optimization & 1 & 5.274 & 5.274 & 8.860 & 0.006 \\
\hline Roots & 1 & 0.735 & 0.735 & 1.230 & 0.275 \\
\hline 2-Way Interactions & 10 & 9.786 & 0.979 & 1.640 & 0.140 \\
\hline Time_step*Objectives & 1 & 1.096 & 1.096 & 1.840 & 0.185 \\
\hline Time_step*Candidates & 1 & 0.697 & 0.697 & 1.170 & 0.288 \\
\hline Time_step* $*$ Optimization & 1 & 3.586 & 3.586 & 6.020 & 0.020 \\
\hline Time_step*Roots & 1 & 0.575 & 0.575 & 0.970 & 0.333 \\
\hline Objectives $*$ Candidates & 1 & 0.395 & 0.395 & 0.660 & 0.421 \\
\hline Objectives*Optimization & 1 & 1.635 & 1.635 & 2.750 & 0.108 \\
\hline Objectives*Roots & 1 & 0.801 & 0.801 & 1.350 & 0.255 \\
\hline Candidates*Optimization & 1 & 0.522 & 0.522 & 0.880 & 0.356 \\
\hline Candidates*Roots & 1 & 0.015 & 0.015 & 0.020 & 0.876 \\
\hline Optimization*Roots & 1 & 0.464 & 0.464 & 0.780 & 0.384 \\
\hline 3-Way Interactions & 10 & 3.644 & 0.364 & 0.610 & 0.792 \\
\hline Time_step*Objectives*Candidates & 1 & 0.475 & 0.475 & 0.800 & 0.379 \\
\hline Time_step*Objectives*Optimization & 1 & 1.025 & 1.025 & 1.720 & 0.199 \\
\hline Time_step*Objectives*Roots & 1 & 0.581 & 0.581 & 0.980 & 0.331 \\
\hline Time_step*Candidates*Optimization & 1 & 0.372 & 0.372 & 0.620 & 0.435 \\
\hline Time_step*Candidates*Roots & 1 & 0.000 & 0.000 & 0.000 & 0.980 \\
\hline Time_step*Optimization*Roots & 1 & 0.664 & 0.664 & 1.120 & 0.299 \\
\hline Objectives $*$ Candidates $*$ Optimization & 1 & 0.296 & 0.296 & 0.500 & 0.486 \\
\hline Objectives $*$ Candidates $*$ Roots & 1 & 0.025 & 0.025 & 0.040 & 0.839 \\
\hline Objectives*Optimization*Roots & 1 & 0.172 & 0.172 & 0.290 & 0.595 \\
\hline Candidates*Optimization*Roots & 1 & 0.034 & 0.034 & 0.060 & 0.813 \\
\hline 4-Way Interactions & 5 & 0.861 & 0.172 & 0.290 & 0.915 \\
\hline Time_step*Objectives*Candidates*Optimization & 1 & 0.437 & 0.437 & 0.730 & 0.398 \\
\hline Time_step*Objectives*Candidates*Roots & 1 & 0.028 & 0.028 & 0.050 & 0.831 \\
\hline Time_step*Objectives*Optimization*Roots & 1 & 0.267 & 0.267 & 0.450 & 0.508 \\
\hline Time_step*Candidates*Optimization*Roots & 1 & 0.073 & 0.073 & 0.120 & 0.728 \\
\hline Objectives*Candidates*Optimization*Roots & 1 & 0.056 & 0.056 & 0.090 & 0.761 \\
\hline 5-Way Interaction & 1 & 0.003 & 0.003 & 0.010 & 0.944 \\
\hline Time_step*Objectives*Candidates*Optimization*Roots & 1 & 0.003 & 0.003 & 0.010 & 0.944 \\
\hline Error & 31 & 18.457 & 0.595 & & \\
\hline Total & 63 & 84.226 & & & \\
\hline
\end{tabular}


Table S10. ANOVA table for Experiment 2 for the improvements in NSE $E_{1}$ with respect to the default model. DF: degrees of freedom; Adj SS: adjusted sum of squares; Adj MS: adjusted mean sum of squares; Candidates: $n$; Roots: $w_{\text {root }}$.

\begin{tabular}{|c|c|c|c|c|c|}
\hline Source & DF & Adj SS & Adj MS & F-Value & P-Value \\
\hline Model & 32 & 39.898 & 1.247 & 13.330 & 0.000 \\
\hline Scenario & 1 & 0.105 & 0.105 & 1.120 & 0.298 \\
\hline Linear & 5 & 30.924 & 6.185 & 66.140 & 0.000 \\
\hline Time_step & 1 & 23.271 & 23.271 & 248.860 & 0.000 \\
\hline Objectives & 1 & 3.714 & 3.714 & 39.720 & 0.000 \\
\hline Candidates & 1 & 0.208 & 0.208 & 2.220 & 0.146 \\
\hline Optimization & 1 & 2.923 & 2.923 & 31.260 & 0.000 \\
\hline Roots & 1 & 0.807 & 0.807 & 8.630 & 0.006 \\
\hline 2-Way Interactions & 10 & 6.188 & 0.619 & 6.620 & 0.000 \\
\hline Time_step*Objectives & 1 & 0.836 & 0.836 & 8.930 & 0.005 \\
\hline Time_step*Candidates & 1 & 0.128 & 0.128 & 1.360 & 0.252 \\
\hline Time_step*Optimization & 1 & 2.229 & 2.229 & 23.840 & 0.000 \\
\hline Time_step*Roots & 1 & 0.665 & 0.665 & 7.110 & 0.012 \\
\hline Objectives $*$ Candidates & 1 & 0.022 & 0.022 & 0.230 & 0.635 \\
\hline Objectives*Optimization & 1 & 1.077 & 1.077 & 11.520 & 0.002 \\
\hline Objectives*Roots & 1 & 0.651 & 0.651 & 6.960 & 0.013 \\
\hline Candidates*Optimization & 1 & 0.079 & 0.079 & 0.850 & 0.364 \\
\hline Candidates*Roots & 1 & 0.002 & 0.002 & 0.020 & 0.900 \\
\hline Optimization*Roots & 1 & 0.500 & 0.500 & 5.350 & 0.028 \\
\hline 3-Way Interactions & 10 & 2.244 & 0.224 & 2.400 & 0.030 \\
\hline Time_step*Objectives*Candidates & 1 & 0.050 & 0.050 & 0.530 & 0.470 \\
\hline Time_step*Objectives*Optimization & 1 & 0.825 & 0.825 & 8.820 & 0.006 \\
\hline Time_step*Objectives*Roots & 1 & 0.530 & 0.530 & 5.660 & 0.024 \\
\hline Time_step*Candidates*Optimization & 1 & 0.046 & 0.046 & 0.500 & 0.487 \\
\hline Time_step*Candidates*Roots & 1 & 0.001 & 0.001 & 0.010 & 0.942 \\
\hline Time_step*Optimization*Roots & 1 & 0.566 & 0.566 & 6.050 & 0.020 \\
\hline Objectives*Candidates*Optimization & 1 & 0.014 & 0.014 & 0.150 & 0.703 \\
\hline Objectives*Candidates*Roots & 1 & 0.000 & 0.000 & 0.000 & 0.963 \\
\hline Objectives*Optimization*Roots & 1 & 0.211 & 0.211 & 2.250 & 0.143 \\
\hline Candidates*Optimization*Roots & 1 & 0.003 & 0.003 & 0.030 & 0.867 \\
\hline 4-Way Interactions & 5 & 0.412 & 0.082 & 0.880 & 0.506 \\
\hline Time_step*Objectives $*$ Candidates*Optimization & 1 & 0.030 & 0.030 & 0.320 & 0.576 \\
\hline Time_step*Objectives*Candidates*Roots & 1 & 0.000 & 0.000 & 0.000 & 0.992 \\
\hline Time_step*Objectives*Optimization*Roots & 1 & 0.290 & 0.290 & 3.100 & 0.088 \\
\hline Time_step*Candidates*Optimization*Roots & 1 & 0.016 & 0.016 & 0.170 & 0.685 \\
\hline Objectives*Candidates*Optimization*Roots & 1 & 0.076 & 0.076 & 0.810 & 0.374 \\
\hline 5-Way Interaction & 1 & 0.026 & 0.026 & 0.280 & 0.603 \\
\hline Time_step*Objectives*Candidates*Optimization*Roots & 1 & 0.026 & 0.026 & 0.280 & 0.603 \\
\hline Error & 31 & 2.899 & 0.094 & & \\
\hline Total & 63 & 42.797 & & & \\
\hline
\end{tabular}


Table S11. ANOVA table for Experiment 2 for the improvements in MARE with respect to the default model. DF: degrees of freedom; Adj SS: adjusted sum of squares; Adj MS: adjusted mean sum of squares; Candidates: $n$; Rgots: $w$

\begin{tabular}{|c|c|c|c|c|c|}
\hline Source & DF & Adj SS & Adj MS & F-Value & P-Value \\
\hline Model & 32 & 27.446 & 0.858 & 16.570 & 0.000 \\
\hline Scenario & 1 & 2.191 & 2.191 & 42.320 & 0.000 \\
\hline Linear & 5 & 18.024 & 3.605 & 69.630 & 0.000 \\
\hline Time_step & 1 & 13.747 & 13.747 & 265.540 & 0.000 \\
\hline Objectives & 1 & 1.943 & 1.943 & 37.530 & 0.000 \\
\hline Candidates & 1 & 0.254 & 0.254 & 4.910 & 0.034 \\
\hline Optimization & 1 & 1.567 & 1.567 & 30.270 & 0.000 \\
\hline Roots & 1 & 0.513 & 0.513 & 9.910 & 0.004 \\
\hline 2-Way Interactions & 10 & 5.046 & 0.505 & 9.750 & 0.000 \\
\hline Time_step*Objectives & 1 & 1.148 & 1.148 & 22.180 & 0.000 \\
\hline Time_step*Candidates & 1 & 0.186 & 0.186 & 3.600 & 0.067 \\
\hline Time_step*Optimization & 1 & 1.448 & 1.448 & 27.970 & 0.000 \\
\hline Time_step*Roots & 1 & 0.502 & 0.502 & 9.700 & 0.004 \\
\hline Objectives*Candidates & 1 & 0.108 & 0.108 & 2.090 & 0.159 \\
\hline Objectives*Optimization & 1 & 1.001 & 1.001 & 19.320 & 0.000 \\
\hline Objectives*Roots & 1 & 0.223 & 0.223 & 4.300 & 0.047 \\
\hline Candidates $*$ Optimization & 1 & 0.118 & 0.118 & 2.270 & 0.142 \\
\hline Candidates*Roots & 1 & 0.005 & 0.005 & 0.100 & 0.756 \\
\hline Optimization*Roots & 1 & 0.308 & 0.308 & 5.950 & 0.021 \\
\hline 3-Way Interactions & 10 & 1.880 & 0.188 & 3.630 & 0.003 \\
\hline Time_step*Objectives*Candidates & 1 & 0.145 & 0.145 & 2.800 & 0.105 \\
\hline Time_step*Objectives*Optimization & 1 & 0.910 & 0.910 & 17.580 & 0.000 \\
\hline Time_step*Objectives*Roots & 1 & 0.201 & 0.201 & 3.880 & 0.058 \\
\hline Time_step*Candidates*Optimization & 1 & 0.117 & 0.117 & 2.260 & 0.143 \\
\hline Time_step*Candidates*Roots & 1 & 0.008 & 0.008 & 0.150 & 0.703 \\
\hline Time_step*Optimization*Roots & 1 & 0.294 & 0.294 & 5.690 & 0.023 \\
\hline Objectives*Candidates*Optimization & 1 & 0.112 & 0.112 & 2.170 & 0.151 \\
\hline Objectives*Candidates*Roots & 1 & 0.016 & 0.016 & 0.310 & 0.580 \\
\hline Objectives*Optimization $*$ Roots & 1 & 0.064 & 0.064 & 1.240 & 0.274 \\
\hline Candidates*Optimization*Roots & 1 & 0.012 & 0.012 & 0.230 & 0.638 \\
\hline 4-Way Interactions & 5 & 0.253 & 0.051 & 0.980 & 0.448 \\
\hline Time_step*Objectives*Candidates*Optimization & 1 & 0.100 & 0.100 & 1.930 & 0.175 \\
\hline Time_step*Objectives*Candidates*Roots & 1 & 0.009 & 0.009 & 0.170 & 0.686 \\
\hline Time_step*Objectives*Optimization*Roots & 1 & 0.063 & 0.063 & 1.220 & 0.278 \\
\hline Time_step*Candidates*Optimization*Roots & 1 & 0.016 & 0.016 & 0.300 & 0.586 \\
\hline Objectives*Candidates*Optimization*Roots & 1 & 0.066 & 0.066 & 1.270 & 0.269 \\
\hline 5-Way Interaction & 1 & 0.053 & 0.053 & 1.020 & 0.321 \\
\hline Time_step*Objectives*Candidates*Optimization*Roots & 1 & 0.053 & 0.053 & 1.020 & 0.321 \\
\hline Error & 31 & 1.605 & 0.052 & & \\
\hline Total & 63 & 29.051 & & & \\
\hline
\end{tabular}


Table S12. ANOVA table for Experiment 3 for the improvements in NSE $E_{2}$ with respect to the default model. DF: degrees of freedom; Adj SS: adjusted sum of squares; Adj MS: adjusted mean sum of squares; Samples: $p_{\text {samp }}$.

\begin{tabular}{lrrrrr}
\hline Source & DF & Adj SS & Adj MS & F-Value & P-Value \\
\hline Model & 24 & 7.368 & 0.307 & 1.710 & 0.102 \\
$\quad$ Scenario & 1 & 3.551 & 3.551 & 19.770 & 0.000 \\
Linear & 7 & 2.313 & 0.330 & 1.840 & 0.128 \\
$\quad$ Time_step & 5 & 2.310 & 0.462 & 2.570 & 0.055 \\
Samples & 1 & 0.002 & 0.002 & 0.010 & 0.915 \\
$\quad$ Greed & 1 & 0.000 & 0.000 & 0.000 & 0.973 \\
2-Way Interactions & 11 & 1.483 & 0.135 & 0.750 & 0.682 \\
$\quad$ Time_step*Samples & 5 & 0.144 & 0.029 & 0.160 & 0.975 \\
Time_step*Greed & 5 & 1.321 & 0.264 & 1.470 & 0.238 \\
$\quad$ Samples*Greed & 1 & 0.019 & 0.019 & 0.110 & 0.748 \\
3-Way Interactions & 5 & 0.021 & 0.004 & 0.020 & 1.000 \\
$\quad$ Time_step*Samples*Greed & 5 & 0.021 & 0.004 & 0.020 & 1.000 \\
Error & 23 & 4.132 & 0.180 & & \\
Total & 47 & 11.500 & & & \\
\hline
\end{tabular}

5 Table S13. ANOVA table for Experiment 3 for the improvements in NSE $E_{1}$ with respect to the default model. DF: degrees of freedom; Adj SS: adjusted sum of squares; Adj MS: adjusted mean sum of squares; Samples: $\boldsymbol{p}_{\text {samp }}$.

\begin{tabular}{lrrrrr}
\hline Source & DF & Adj SS & Adj MS & F-Value & P-Value \\
\hline Model & 24 & 2.158 & 0.090 & 2.290 & 0.026 \\
$\quad$ Scenario & 1 & 0.000 & 0.000 & 0.000 & 0.971 \\
Linear & 7 & 1.644 & 0.235 & 5.980 & 0.000 \\
$\quad$ Time_step & 5 & 1.625 & 0.325 & 8.270 & 0.000 \\
Samples & 1 & 0.017 & 0.017 & 0.440 & 0.514 \\
$\quad$ Greed & 1 & 0.002 & 0.002 & 0.040 & 0.843 \\
2-Way Interactions & 11 & 0.501 & 0.046 & 1.160 & 0.366 \\
$\quad$ Time_step*Samples & 5 & 0.156 & 0.031 & 0.790 & 0.564 \\
Time_step*Greed & 5 & 0.344 & 0.069 & 1.750 & 0.163 \\
$\quad$ Samples*Greed & 1 & 0.000 & 0.000 & 0.000 & 0.999 \\
3-Way Interactions & 5 & 0.013 & 0.003 & 0.070 & 0.997 \\
$\quad$ Time_step*Samples*Greed & 5 & 0.013 & 0.003 & 0.070 & 0.997 \\
Error & 23 & 0.904 & 0.039 & & \\
Total & 47 & 3.062 & & & \\
\hline
\end{tabular}


Table S14. ANOVA table for Experiment 3 for the improvements in MARE with respect to the default model. DF: degrees of freedom; Adj SS: adjusted sum of squares; Adj MS: adjusted mean sum of squares; Sammples: $p$

\begin{tabular}{lrrrrr}
\hline Source & DF & Adj SS & Adj MS & F-Value & P-Value \\
\hline Model & 24 & 1.438 & 0.060 & 6.780 & 0.000 \\
$\quad$ Scenario & 1 & 0.643 & 0.643 & 72.820 & 0.000 \\
Linear & 7 & 0.630 & 0.090 & 10.190 & 0.000 \\
$\quad$ Time_step & 5 & 0.605 & 0.121 & 13.700 & 0.000 \\
$\quad$ Samples & 1 & 0.021 & 0.021 & 2.320 & 0.141 \\
Greed & 1 & 0.005 & 0.005 & 0.530 & 0.473 \\
2-Way Interactions & 11 & 0.152 & 0.014 & 1.570 & 0.175 \\
$\quad$ Time_step*Samples & 5 & 0.115 & 0.023 & 2.610 & 0.052 \\
Time_step*Greed & 5 & 0.033 & 0.007 & 0.760 & 0.590 \\
Samples*Greed & 1 & 0.004 & 0.004 & 0.430 & 0.519 \\
3-Way Interactions & 5 & 0.012 & 0.002 & 0.270 & 0.925 \\
$\quad$ Time_step*Samples*Greed & 5 & 0.012 & 0.002 & 0.270 & 0.925 \\
Error & 23 & 0.203 & 0.009 & & \\
Total & 47 & 1.642 & & & \\
\hline
\end{tabular}

